\title{
The Classification of Extragalactic X-ray Jets
}

\author{
D. E. Harris*, F. Massaro* and C. C. Cheung ${ }^{\dagger}$ \\ ${ }^{*}$ SAO, 60 Garden St., Cambridge, MA 02138, USA \\ ${ }^{\dagger} N R L / N R C, 4555$ Overlook Ave SW, Washington, DC, 20375 USA
}

\begin{abstract}
The overall classification of X-ray jets has clung to that prevalent in the radio: FRI vs. FRII (including quasars). Indeed, the common perception is that X-ray emission from FRI's is synchrotron emission whereas that from FRII's may be IC/CMB and/or synchrotron. Now that we have a sizable collection of sources with detected X-ray emission from jets and hotspots, it seems that a more unbiased study of these objects could yield additional insights on jets and their $\mathrm{X}$-ray emission. The current contribution is a first step in the process of analyzing all of the relevant parameters for each detected component for the sources collected in the XJET website. This initial effort involves measuring the ratio of X-ray to radio fluxes and evaluating correlations with other jet parameters. For single zone synchrotron X-ray emission, we anticipate that larger values of $\mathrm{fx} / \mathrm{fr}$ should correlate inversely with the average magnetic field strength (if the acceleration process is limited by loss time equals acceleration time). Beamed IC/CMB X-rays should produce larger values of $\mathrm{fx} / \mathrm{fr}$ for smaller values of the angle between the jet direction and the line of sight but will also be affected by the low frequency radio spectral index.
\end{abstract}

Keywords: relativistic jets

PACS: $98.62 . J s$

\section{DEFINING THE PROBLEM}

The paradigm for the classification of extragalactic jets is based on the Fanaroff-Riley ([2]) distinction between powerful FRII radio galaxies (and radio quasars) on the one hand, and low power FRI radio galaxies (and BL Lac objects) on the other. The (perhaps overly simplified) notion is that the jets of FRI sources are lossy, often displaying a number of brightness enhancements (a.k.a. "knots") whereas FRII jets in radio galaxies ("RG" hereafter) are often difficult to detect and deposit the bulk of their energy at large distances from the parent galaxy in the radio 'hotspots'. Generally speaking, FRI jets often terminate within the confines of their host galaxy (e.g. M87), or at least the well collimated section of the jets are of similar size as their host (e.g. 3C 31).

Obviously this simple interpretation has a number of caveats; chief amongst them is the effect of relativistic beaming which means that we must make model-based assumptions to imagine what the jets of Cyg A would look like if one of them was close to our line of sight (l.o.s.). In the unification hypothesis ([7]) radio quasars and FRII RG are distinguished solely on the basis of their viewing angle. If we were to view the jet of Cyg A close to the l.o.s. would it be a knotty jet like that of $3 \mathrm{C} 273$ ?

Our primary interest in this problem arises from the question of the emission processes for the generation of X-rays from jets. The idea that kpc scale jets have substantial bulk Lorentz factors, $\Gamma \gtrsim 5-10$ that would engender copious production of X-rays from inverse Compton scattering of low energy relativistic electrons on photons of the cosmic microwave background ("IC/CMB", $[5,1,3]$ ) came to be the accepted explanation for 
X-ray emission from quasar and FRII RG jets. For FRI jets, synchrotron emission from very high energy electrons (i.e. electrons with Lorentz factors $\gamma \geq 10^{7}$ ) is thought to provide a consistent suite of physical parameters and simple models were able to produce the observed spectral energy distributions (SED).

Thus the classification of X-ray emitting jets tended to follow the FRI - FRII separation, with the additional notion that FRI jets would generally have the X-ray spectral index $\left(\alpha_{x}\right.$, defined by flux density, $\left.\mathrm{S}_{v} \propto v^{-\alpha}\right)>1$ whereas $\alpha_{x}$ for quasars and FRII RG's would be $<1$. These values of $\alpha_{x}$ are consistent with expectations that at the high end of the electron distribution $(\mathrm{N}(\gamma))$, we may expect steep spectra because we may be observing close to an exponential cutoff and/or the initial $\mathrm{N}(\gamma)$ has been strongly affected at these energies by synchrotron and IC losses which go as $\gamma^{2}$; and at the low end of $\mathrm{N}(\gamma)$, the emitted spectra (either synchrotron or IC) would be flatter.

A number of doubts and counter examples have been raised about the IC/CMB model of X-ray emission of quasar and FRII jets. Amongst these are: (a) the inability to independently demonstrate that $\Gamma \geq 5$ on kpc scales; (b) the assumption that one can extrapolate $\mathrm{N}(\gamma)$ from the radio regime $\left(\gamma \approx 10^{4}\right)$ down to $\gamma \approx 100$ with a power law of slope $-\mathrm{p}$ with $\mathrm{p}=2 \times \alpha_{\text {radio }}+1$ (i.e. are there really enough low energy electrons?); and (c) the evidence that the X-ray jet of 3C 273 comes from a second synchrotron component rather than IC/CMB ([6]).

These concerns have prompted us to attempt to devise a different classification scheme for X-ray jets, hoping thereby to achieve a better understanding of the role of IC and synchrotron emissions at X-ray frequencies. The present contribution reports on the first step of this project: an investigation of the ratio of X-ray to radio flux for X-ray detected knots and hotspots. To do this, we have embarked on a standard reduction scheme of all $\mathrm{X}$-ray jets detected by the CXO (Chandra X-ray Observatory). The finding list for these sources comes from the XJET compilation ${ }^{1}$, see Massaro et al., this volume.

\section{PRELIMINARY RESULTS}

Our initial focus is an investigation of trends and correlations involving the parameter

$\mathrm{R}=\frac{f_{x}}{f_{r}}$, the ratio of the $\mathrm{X}$-ray flux to the radio flux. The former comes from our flux map photometry (0.5-7 KeV, with no correction for absorption) and the latter is calculated as $v S_{v}$. These radio fluxes come from VLA, MERLIN, and ATCA maps at frequencies between 1.4 and $8 \mathrm{GHz}$.

In order to visualize how $\mathrm{R}$ may depend on various parameters, consider a generalized electron spectrum consisting of 3 power laws (fig. 1, left panel): the lowest energy segment characterized by an amplitude $\mathrm{N}_{1}$, power law index $\mathrm{p}_{1}$, covering the range of $\gamma$ 's from the tens to hundreds. The next segment, $\mathrm{N}_{2}$ with a somewhat steeper spectrum would cover the energy range responsible for the normal radio band via synchrotron emission. The third segment would cover the high end, $\gamma=10^{6}$ to $10^{8}$. The number of electrons contributing to IC/CMB is meant to be represented by $\mathrm{N}_{1}$; the radio synchrotron by $\mathrm{N}_{2}$, and the X-ray synchrotron by $\mathrm{N}_{3}$. Obviously the relative values

${ }^{1}$ http://hea-www.harvard.edu/XJET/ 
Electron Spectrum, N(E)

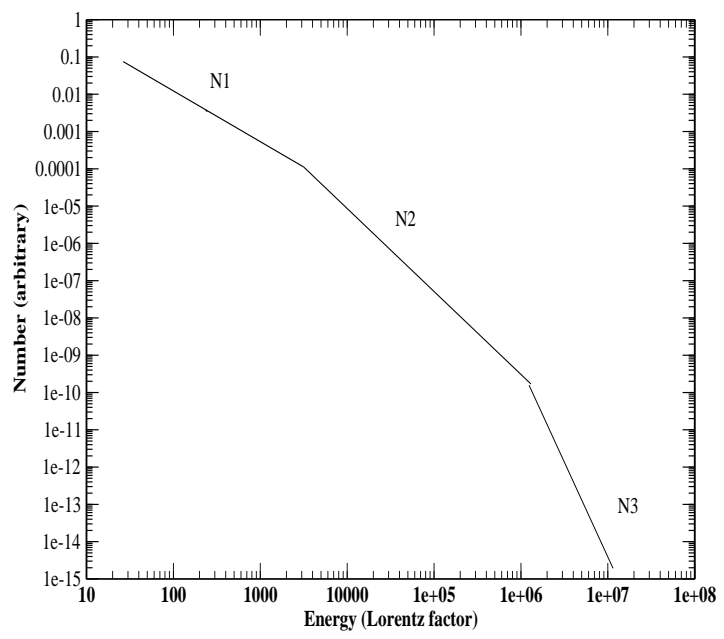

Flux Ratio vs. X-ray Luminosity

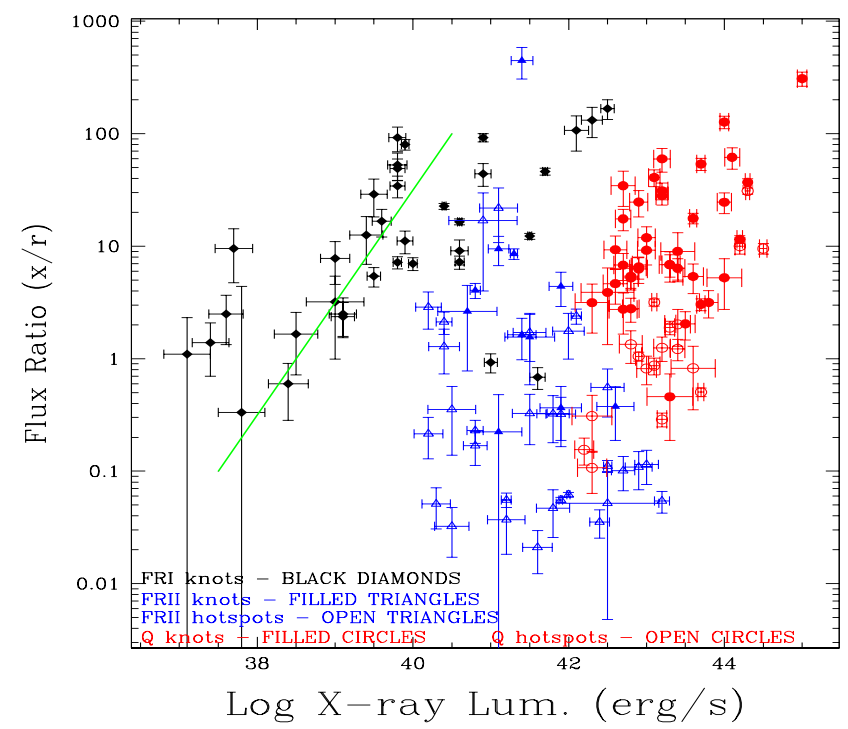

FIGURE 1. Left: An idealized, broken power law electron spectrum. N1 represents the number of electrons contributing to IC/CMB X-rays; N2 the number contributing to the radio emission; and N3, the number responsible for synchrotron X-rays. Right: A scatter plot of R versus X-ray luminosity $\left(\Omega_{\Lambda}=0.73\right.$, $\Omega_{M}=0.23$, and $H_{0}=71 \mathrm{~km} \mathrm{~s}^{-1} \mathrm{Mpc}^{-1}$ ). FRI knots are black diamonds; FRII knots are blue filled triangles, and quasar knots are red filled circles. Hotspots are open triangles (blue FRII) and circles (red Q). The green line is not a fit to anything; it has a slope of +1 (see text).

of the N's depends on the three p's, the energies of any low and/or high energy cutoffs, and the energies of the spectral breaks.

Considering the emission produced by this electron distribution, the parameters relevant to the value of $\mathrm{R}$ can be isolated by:

$$
R=\frac{X(i c)+X(\text { sync })}{\text { radio }(\text { sync })}
$$

and for the case of beamed IC/CMB X-rays, of interest here,

$$
R \propto \frac{f(\theta) N_{1} u(v)+N_{3} B^{2}}{N_{2} B^{2}} \propto \frac{N_{1}}{N_{2}} \frac{f(\theta) \Gamma^{2}}{B^{2}}+\frac{N_{3}}{N_{2}}
$$

where $f(\theta)$ is the extra beaming factor for $\operatorname{IC/CMB~}([3,4]) ; \theta$ is the angle between the jet and the line of sight; $u(v)$ is the photon energy density; $\Gamma$ is the bulk Lorentz factor of the jet; and B is the average magnetic field strength. For FRI knots, the second term is thought to be the dominating contributor so R is determined by the ratio of N's, which, in turn depends on $\alpha_{2}, \alpha_{3}$, and the frequency of the break as well as the frequency of an exponential cutoff, if relevant. If the X-ray emission of quasar knots (and hotspots) is primarily $\mathrm{IC} / \mathrm{CMB}$, then the first term dominates and there is a significantly larger number of (unknown) parameters. In addition to the corresponding spectral parameters of the synchrotron case for the ratio of N's, we have the unknown angle to the l.o.s., the 
unknown value of $\Gamma$, and the magnetic field strength. A priori, it might seem that one should not expect to find any significant correlation between $\mathrm{R}$ and other jet parameters.

We note that while the effect of the magnetic field strength is explicit in the case of $\mathrm{IC} / \mathrm{CMB}$, it is also implicitly present for the synchrotron case since a stronger field will usually produce a lower frequency for the high energy cutoff as well as increasing the value of $\alpha_{3}$. Thus in general, we may expect stronger field regions to have lower values of R. (than would otherwise be the case).

Because of space limitations, we show only one of our first set of plots relating $\mathrm{R}$ to other jet properties. The plot shown in the right panel of fig. 1 ( $R$ as a function of $X$-ray luminosity) shows a rather clean separation of FRI, FRII, and Q features.

If all features had the same one-to-one correspondence between X-ray flux and radio flux, then all points would lie on a horizontal line. The line in the figure has a slope of +1 and is shown to demonstrate the locus of points which would describe knots that had the same radio flux but different X-ray fluxes. Obviously we do not expect either of these conditions so we are surprised to find the majority of FRI knots clustered about a particular +1 line. Although the quasar distribution follows the same trend, this behavior is not evident for the FRII hotspots.

\section{SUMMARY}

From our preliminary analysis using the ratio of X-ray to radio fluxes, we have found that hotspots tend to have a lower ratio than knots. Features classified as 'quasar hotspots' generally have smaller values of $\mathrm{R}$ than quasar knots, and most FRII hotspots have smaller R values than FRI and quasar knots. Most FRI and quasar knots have R values between 1 and 100. We have failed to find any significant distinction between FRI and quasar knots except for the obvious difference in apparent $\mathrm{x}$-ray luminosity.

\section{ACKNOWLEDGMENTS}

We are indebted to a number of colleagues who have generously donated radio maps for our use, and for public access via the XJET webpage. Other radio maps were downloaded from the NRAO VLA Archive Survey. The work at SAO was partially supported by NASA grant AR6-7013X. FM acknowledges the Foundation BLANCEFLOR Boncompagni-Ludovisi, n'ee Bildt for the grant awarded him in 2009.

\section{REFERENCES}

1. A. Celotti, G. Ghisellini, \& M. Chiaberge, MNRAS 321, L1-5 (2001)

2. B. L. Fanaroff \& J. M. Riley, J. M., MNRAS 167, 31p-36p (1974)

3. D. E. Harris and H. Krawczynski, ApJ 565, 244-255 (2002)

4. F. Massaro, D. E. Harris, M. Chiaberge, P. Grandi, F. D. Macchetto, S. A. Baum, C. P. O’Dea, A. Capetti, ApJ 696, 980-985 (2009)

5. F. Tavecchio, L. Maraschi, R. M. Sambruna, \& C. M. Urry, ApJL 544, L23-26 (2000)

6. Y. Uchiyama et al., ApJ 648, 910-921 (2006)

7. C. M. Urry \& P. Padovani, PASP 107, 803-845 (1995) 